Eur Psychiatry Ed. Esp. (1999); 6: 259-261

NOTICIAS DE LA AEP

\title{
Simposio sobre formación en psiquiatría general y psiquiatría del niño y del adolescente
}

\author{
L. A. Sheldon' y P. L. Cornwall \\ 'Community Learning Disability Service, Londres, 'Departamento Universitario de Psiquiatría, \\ Royal Victoria Infirmary, Queen Victoria Road, Newcastle upon Tyne, NE1 4LP, Reino Unido \\ psiquiatría del niño y del adolescente / formación / EFPT
}

\section{INTRODUCCIÓN}

Este simposio sobre formación psiquiátrica fue el primero organizado por una Organización de Psiquiatras en Prácticas Monoespecialistas Europeos: el Foro Europeo de todos los Psiquiatras en Prácticas (EFPT). El EFPT se fundó en 1992 y se reúne cada año en un país diferente $[1,2]$. La afiliación nacional ha crecido sin parar e incluye ahora organizaciones de psiquiatras en prácticas de todas las naciones de la Unión Europea, así como Suiza y la participación como observadores en 1998 de Croacia, República Checa, Estonia, Hungría, Israel, Macedonia y Polonia. Los congresos del EFPT han debatido todos los aspectos de la formación y han participado en el desarrollo de normas de formación con las Juntas de la UEMS (Union Européenne des Médecins Spécialistes) de Psiquiatría y Psiquiatría del Niño y del Adolescente.

El simposio tuvo lugar en la Universidad de Antwerp en abril de 1998, y asistieron a él todos los delegados nacionales al EFPT inmediatamente antes de su reunión anual en Gante. También estuvieron presentes psiquiatras en prácticas y formadores de toda Europa y los Estados Unidos. La jornada se dividió en tres áreas temáticas: enseñanza y supervisión, evaluación de la calidad y armonización de la formación en Europa.

\section{Enseñanza y supervisión}

El Dr. Kaltiala-Heino, presidente electo del EFPT, presentó datos sobre un estudio de los psiquiatras en prácticas en Finlandia sobre la competencia que ellos anticipaban para sí al final de su formación. Las competencias esperadas más altas eran en psicofarmacología y en evaluación psiquiátrica, mientras que las más bajas estaban en administración y enseñanza y supervisión. La competencia esperada en psicoterapia era sorprendentemente baja. El profesor Compernolle (Universidad de Amsterdam) puso de relieve el valor de la observación y supervisión en vivo de los psiquiatras en prácticas en el trabajo con los pacientes como la piedra angular de la formación psiquiátrica. Puso en duda la idea que él dijo que existía en algunos países de que la psicoterapia personal durante la formación proporciona la garantía esencial de que los psiquiatras recién titulados serán efectivos clínicamente. Un presiden-

Sheldon LA, Cornwall PL. Symposium: training in general psychiatry and child and adolescent psychiatry. Eur Psychiatry 1998; $13: 379-80$. 
te anterior del EFPT, el Dr. L. Hansen, describió un innovador modelo de orientación clínica diaria pàra psiquiatras en prácticas en Roskilde, Dinamarca. Puso de relieve la distinción entre la orientación cotidiana y las cualidades mentoras de la supervisión, que incluye apoyo moral, orientación de carrera y proporcionar visión y perspectiva. Todos los oradores hicieron hincapié en la necesidad de una supervisión regular, amplia y de alta calidad para los psiquiatras en prácticas. Esto no era lo menos importante de manera que pudieran convertirse en supervisores satisfactorios una vez titulados.

\section{Evaluación de la calidad}

Se acepta por lo general que establecer normas en la formación es más sencillo que asegurar su cumplimiento efectivo. El Dr. Cornwall describió la reciente introducción en el Reino Unido de un diario de formación psiquiátrica cuyo formato se ajusta a las recomendaciones de la Junta de la UEMS de Psiquiatría. Los diarios parecen funcionar mejor cuando son propiedad del psiquiatra en prácticas, se utilizan para establecer objetivos educativos y ayudan a la supervisión, pero no se percibe que sean un método para su evaluación. El Dr. Sheldon describió el procedimiento establecido desde hace largo tiempo en el Reino Unido e Irlanda de visitas de inspección a los esquemas de formación psiquiátrica por el Real Colegio de Psiquiatras. Todos los equipos de inspección, de tres miembros, incluyen un psiquiatra en prácticas finalizando su formación cuyo rol especificado es hablar con cada psiquiatra en prácticas individualmente. La opinión de éste se considera esencial y de igual peso que el de los visitantes formadores. El Dr. Scully, Presidente del Comité de Educación Médica de la Asociación Psiquiátrica Americana, describió el examen de residentes psiquiátricos en formación (PRITE) en uso en la actualidad en los Estados Unidos desde hace más de diez años. Este examen no se utiliza para fines de certificación, pero permite a los psiquiatras en prácticas evaluar sus propios progresos sobre una base anual [3]. Del mismo modo, los programas de formación pueden comparar la calidad de la formación que ofrecen frente a otros programas sobre la base de los resultados anuales del PRITE.

\section{Armonización de la formación en Europa}

El deseo de armonizar la formación psiquiátrica en Europa plantea difíciles preguntas, porque la psiquiatría general y la psiquiatría del niño y del adolescente se practican de manera muy diferente en los diversos países. El profesor GómezBeneyto, presidente de la Junta de la UEMS de Psiquiatría, estableció de manera destacada que las similitudes en la práctica psiquiátrica a través de Europa eran mayores que las diferencias. Puso de relieve que armonización no significaba uniformidad, sino más bien asegurar normas de calidad y afrontamiento de las diferencias de una manera positiva. Defendió facilitar el intercambio de psiquiatras en prácticas entre países, la idea de un diario de formación europeo y equipos de inspección europea para visitar los esquemas de formación. El Dr. von Salis, Presidente de la Junta UEMS de Psiquiatría del Niño y del Adolescente, comentó que varios países europeos no reconocen la psiquiatria del niño y del adolescente como una especialidad independiente, mientras que en los países que la reconocen, la duración de la formación puede variar de dos a siete años. Los requisitos mínimos de la Junta de la UEMS han recomendado cinco años de formación de graduados. El Dr. von Salis prosiguió señalando que el contenido de la formación varía considerablemente: algunos países ofrecen métodos enteramente biológicos y conductuales de tratamiento, mientras que en el otro extrémo, otros ofrecen exclusivamente enfoques psicodinámicos.

\section{CONCLUSIÓp!}

Este simposio de un día de duración reunió a representantes de las organizaciones nacionales de psiquiatras en prácticas, formadores y oradores de toda Europa. Se cubrieron áreas clave en el desarrollo y la mejora de la formación psiquiátrica. El debate fue de gran amplitud, tanto dentro como fuera de la sala de conferencias, y se forjaron vínculos entre psiquiatras en prácticas y profesionales que traspasaban los límites nacionales. Una experiencia común para muchos fue la realidad de intentar mejorar la formación en un clima de recortes presupuestarios de la salud 
mental barriendo Europa y la aplicación de una cultura comercial a los sistemas de asistencia sanitaria. Se acordó que se podría apoyar el mantenimiento del impulso para mejorar las normas de formación por el intercambio mutuo de información, la implicación de los psiquiatras en prâcticas en todos los niveles de toma de decisiones y la creación de normas básicas para toda Europa. Este simposio fue quizá una piedra angular hacia este objetivo.

\section{BIBLIOGRAFÍA}

1 Cornwall PL, Gribbin N. The Fifth European Forum for all Psychiatric Trainees. Eur Psychiatry 1998; 13: 53.

2 Cornwall PL, Sheldom L. The Fourth European Trainees Forum. Psychiatric Bull R Coll Psychiatr 1997; 21: 117.

3 Webb LC, Juul D, Reynolds CF 3rd, Ruiz B, Ruiz P, Scheiber SC et al. How well does the psychiatry residency in training exam predict performance on the American Board ol Neurology and Psychiatry Part I. Examination? Am J Psychiatry 1996; 153: 831-2. 